\title{
C. COBENGE \\ REDES DE CONHECIMENTO STEAM NA FORMAÇÃO DO ENGENHEIRO CONTEMPORÂNEO: UMA REVISÃO SISTEMÁTICA DA LITERATURA
}

\author{
DOI: 10.37702/2175-957X.COBENGE.2021.3685
}

Roni Costa Ferreira - ronicostaf@gmail.com

CEFETRJ

RUA São Cristóvão 805

20940-001 - RIO DE JANEIRO - RJ

Marco Antonio Barbosa Braga - marcobraga@namelab.education CEFETRJ

Rua General Goes Monteiro 08

22290-080 - RIO DE JANEIRO - RJ

Resumo: Este artigo mostra a dificuldade de encontrar modelos de aprendizagem STEM que aprimorem o fluxo de informações e o trabalho em rede para a inovação. Então, pretendeu-se elucidar sobre o conceito e a criação de Redes de Conhecimento. Utilizou-se como metodologia de pesquisa uma revisão sistemática da literatura, que recolheu das principais bases científicas 167 artigos para embasar a discussão. A etapa de validação da qualidade recortou 15 artigos para responder as perguntas da investigação. Os resultados apresentam as Redes de Conhecimento como ecossistemas de inteligência coletiva, que surgem na sua maioria como resposta a competitividade e colaboração. Possui como objetivo principal a formação para a inovação.

Palavras-chave: Educação STEM, Redes de Conhecimento, Inovação Colaborativa, Aprendizagem por Projetos 


\section{REDES DE CONHECIMENTO STEAM NA FORMAÇÃO DO ENGENHEIRO CONTEMPORÂNEO: UMA REVISÃO SISTEMÁTICA DA LITERATURA}

\section{INTRODUÇÃO}

$\mathrm{Na}$ conjuntura do mundo contemporâneo, dois fatores se tornaram altamente relevantes para o aprimoramento e mudanças na cultura organizacional e para a manutenção do ecossistema de inovação. Um destes fatores passa pelo entendimento e gerenciamento do fluxo de informações, que as organizações precisam tratar nos seus processos internos e externos. As informações "[...] apoiam o processo decisório, exercem influências sobre o comportamento das pessoas e passam a ser um vetor importantíssimo, pois podem multiplicar a sinergia dos esforços ou anular o resultado obtido pelo conjunto" (MORAES; FADEL, 2007, p.103). Quando o fluxo de informações é bem aproveitado, isto reverbera por todos os níveis da organização, transformando comportamentos, aumentando a produtividade, a qualidade e principalmente, o seu grau de inteligência competitiva (CAVALCANTE; VALENTIM, 2008). Compreender a importância do compartilhamento de informações deve ser aprendida por todos os colaboradores da organização, a fim das discussões, em diferentes grupos, gerarem novas percepções e agregarem valor a um conhecimento coletivo, que melhora o suporte nas tomadas de decisão. Investir em colaboradores, que ao invés de reter, permitem que a informação circule de forma livre e flexível, torna-se crucial para o aumento de projetos inovadores. 
Diante deste contexto, outro fator, que também se destaca em um mundo globalizado e conectado, pelas redes digitais que compõe a Internet, torna-se fundamental para a sobrevivência das organizações: o trabalho em rede. É por meio das redes, suportadas pelas tecnologias de informação e comunicação, que as organizações têm construído um continuum movimento de interação, de relacionamentos, de troca de experiências e conhecimento. $O$ trabalho dentro destes agrupamentos é distribuído entre seus membros, priorizando desta forma, a colaboração em detrimento do velho modelo de competição, hierarquização e centralização do poder (BARABÁSI, 2009). Dentro da nova topologia de trabalho em rede, continuam existindo as lideranças, as discussões, as negociações e os interesses individuais, mas a dinâmica do conviver e do compartilhar mudam a forma de acesso aos recursos, sejam tangíveis ou intangíveis, assim como sua aplicação. Enquanto a filosofia da hierarquização tende para a escassez, a mentalidade e as práticas da rede colaborativa geram múltiplos caminhos, empoderando todos os seus membros e estabelecendo o princípio da confiança. Juntos, estes dois fatores, o fluxo livre e gerenciável de informações e as redes de trabalho colaborativo, formam o que definiu-se nomear neste artigo como "Redes de Conhecimento".

Neste sentido, a formação do engenheiro e demais profissionais de tecnologia necessita acompanhar e se adequar às exigências de uma conjuntura de incertezas, mudanças constantes e problemas cada vez mais complexos. Haja vista, que uma das grandes causas das evasões dos cursos de Engenharia é de ordem pedagógica, principalmente no Ciclo Básico, onde as disciplinas como matemática e ciências são ministradas (GODOY; ALMEIDA, 2020). Outras pesquisas, demonstram que atividades práticas que se aproximem do ambiente profissional, tendem a causar impactos mais positivos na preparação dos engenheiros do futuro (LIMA et al., 2020). Portanto, deve-se buscar abordagens pedagógicas capazes de desenvolver, nos estudantes da área, competências que os empoderem para trabalhar colaborativamente em rede, analisando um grande número de informações, e criando soluções adequadas para enfrentar os desafios do futuro (THOMASSEN; STENTOFT, 2020). Até porque, os próprios postos de trabalho que eles assumirão, ainda estão sendo criados ou não estão previstos (WORLD ECONOMIC FORUM, 2020).

Logo, o objetivo desta pesquisa é o de entender o que são as Redes de Conhecimento e quais são os elementos necessários para sua criação. A fim de alcançar tal objetivo, usou-se uma metodologia que investigou e analisou artigos nas principais bases científicas, mundialmente reconhecidas, buscando por abordagens inovadoras que obtiveram ganhos, mensuráveis e ou observáveis, na comunicação, colaboração, criatividade e na integração entre saberes e projetos STEAM, realizados dentro do processo educativo de engenheiros e outros profissionais do setor tecnológico. Este documento está organizado da seguinte forma: na seção 2 apresenta-se uma fundamentação teórica, seguida pela seção 3 , onde descreve-se a metodologia de pesquisa. Na seção 4 , os resultados da análise, relacionados a questão de pesquisa, e por fim as conclusões.

\section{FUNDAMENTAÇÃO TEÓRICA}

O grande desafio na formação de engenheiros e profissionais de tecnologia é proporcionar uma mudança significativa e profunda no modelo educacional em vigor: "Modelos educacionais unilaterais, monolíticos e lineares relacionados aos processos de ensino-aprendizagem já não conseguem mais responder aos desafios pedagógicos da atualidade, o que vem exigindo a construção de novas abordagens teóricas e tecnológicas mais potentes dedicadas à gestão do conhecimento (CAMPOS, 2011). 
O setor produtivo e o desenvolvimento de cidades inteligentes (smart cities) caminham rapidamente para consolidar a quarta revolução industrial, onde domínios do conhecimento como Big Data, Internet das Coisas, Inteligência Artificial, Robótica, Biotecnologia, Nanotecnologia, entre outros saberes interdisciplinares, se mostram cada vez mais presentes na integração de pessoas, artefatos e ambientes. No Brasil, por exemplo, em recente relatório da Confederação Nacional da Indústria (CNI), destaca-se como uma das dimensões prioritárias em conformidade com as diretrizes da Indústria 4.0, a formação adequada de recursos humanos. Segundo o relatório (CNI, 2016): "A integração de diversas formas de conhecimento, característica desse modo de produção, exigirá equipes multidisciplinares, com elevado nível de conhecimento técnico e com capacidade de interação de diferentes áreas de conhecimento" (p. 29).

Desta forma, é preciso que as instituições educacionais, desde a Educação Básica à Superior, invistam em estratégias pedagógicas, concatenadas com tecnologias interativas, com avaliações mais centradas no processo e uma visão mais holística dos saberes. No decorrer desta transformação, "o educador 4.0 deve ter percepção e flexibilidade para exercer o papel de mediador, orientador e pesquisador para criar novas oportunidades de aprendizagem" (TONINI; PEREIRA, 2019, p. 47). Assim, o movimento STEM surge com a missão de fortalecer uma aprendizagem integrada às transformações da sociedade, e fornecer mudanças na forma de trabalhar conteúdos, habilidades e valores no espaço escolar.

O STEM tem sido adotado como programa educacional governamental em muitos países, como o Estados Unidos (EUA), Canadá, Finlândia, Reino Unido, Austrália e China (BACICH; HOLANDA, 2020). Sua contribuição é percebida na forma como lida com os desafios contemporâneos e desenvolve a criatividade, a colaboração, a comunicação, o engajamento e o pensamento crítico. O acrônimo STEM, do inglês Science, Technology, Engineering and Mathematics, surgiu nos EUA, mais ou menos, na década de 80, e representa a integração entre as áreas de Ciências, Tecnologia, Engenharia e Matemática (PUGLIESE, 2020). O movimento possui três marcas principais: a interdisciplinaridade, o protagonismo discente, e a diversidade. Recentemente, a letra A, do inglês Arts (Artes e Design), foi acrescentada ao acrônimo, ficando STEAM e representando de maneira geral as áreas de humanidades. O STEM ou STEAM representa um ambiente de valorização da criatividade dos estudantes e de construção colaborativa de conhecimento (VEDENPÄÄ, LONKA, 2014; TAN; CHAN; BIELACZYC et al., 2021). Quando aliada a Project Based Learning (PBL), ou aprendizagem por projetos, o desafio proposto, inicialmente ruidoso e desordenado, como a realidade, ganha métodos de gerenciamento, sem perder a flexibilidade, e ainda desenvolve as habilidades profissionais e empreendedoras dos estudantes (ISHA DECOITO, 2016; SÁNCHEZ LUDEÑA, 2019). O impacto deste processo de investigação e resolução de problemas culmina em uma verdadeira "fábrica" de inovação. Segundo Braga (2021):

Ninguém ensina outra pessoa a ser inovadora. Mas isso não impede que se criem condições para que a inovação seja aprendida. Ao se defrontar com problemas e pensar sobre eles buscando soluções, todo estudante é capaz de desenvolver competências ligadas à inovação (p. 21).

Contudo, integrar ciência, tecnologia, engenharia, arte e matemática, continua sendo uma abordagem pedagógica desafiadora e complexa e por isso alguns estudos ainda demonstram a carência de modelos e processos de modelagem de educação STEM autêntica, enraizada na interdisciplinaridade, na prática e na resolução de problemas reais (HALLSTRÖM; SCHÖNBORN, 2019). Este trabalho pretende ser mais um passo para reduzir a lacuna que existe entre os modelos existentes e as premissas de um modelo STEM autêntico, ressaltando as "Redes de Conhecimento" como uma possível solução. 


\section{$3 \quad$ METODOLOGIA DE PESQUISA}

A metodologia adotada foi a Systematic Literature Review(SLR) - revisão sistemática da literatura - seguindo as diretrizes e modelo sistemático de revisão proposto por Kitchenham e Charters (2007). De acordo com estas diretrizes, o processo SLR inclui várias atividades, que podem ser agrupadas em três fases principais: planejamento da SLR, condução da SLR e relatório da SLR. Segundo os autores, a SLR serve para identificar, analisar e interpretar todas as evidências disponíveis relacionadas a uma questão de pesquisa, bem específica, de forma que seja imparcial e repetível.

\subsection{Planejamento da SLR}

A atividade de planejamento da SLR requer dois itens principais. O primeiro diz respeito a necessidade e motivação da pesquisa, que neste caso se configura em: identificar estudos que possam definir as características e singularidades de uma rede de conhecimento, a fim de se propor um modelo de educação STEM autêntico, para o ensino de engenharia e demais profissões da área de tecnologia. No segundo item elencamos as perguntas que nortearão a pesquisa:

- O que é uma rede de conhecimento?

- Como surge uma rede de conhecimento?

- Quais são os objetivos de uma rede de conhecimento?

- Que contribuições uma rede de conhecimento oferece para um processo de inovação?

- Quais são as dificuldades comuns para se manter uma rede de conhecimento ativa?

\subsection{Condução da SLR}

A estratégia de busca incluiu uma seleção automática de artigos nas principais bases científicas globais, utilizando um conjunto de palavras chaves para validar os artigos selecionados. Realizamos buscas piloto para refinar a string de busca de forma iterativa. Excluímos palavras-chave cuja inclusão não retornou documentos adicionais nas buscas automáticas. Após várias iterações, definiu-se a seguinte string de busca usada para pesquisar dentro do título, do resumo e palavras-chave dos artigos:

("network of knowledge" OR "collaborative network" OR "collective intelligence" OR "social network") AND ("engineering education" OR "creative clusters" OR "engineering thinking" OR "innovative environment" OR "makerspaces" OR "STEM education") AND ("innovation education" OR "entrepreneurship" OR "knowledge management").

Outro item desta fase foi a definição dos critérios de inclusão e exclusão para delinear os resultados de acordo com o objetivo da pesquisa, veja Tabela 1:

Tabela 1 - Critérios de Inclusão e Exclusão da Pesquisa

\begin{tabular}{l|l} 
Inclusão & Exclusão
\end{tabular}

Estudos que demonstrem o uso de redes de conhecimento

Estudos que discutem a importância das redes de conhecimento

Estudos que explicam o conceito de redes de conhecimento
Estudos duplicados

Literatura cinza (estudos secundários, relatórios técnicos, manuais, teses e dissertações)

Estudos fora do escopo da pesquisa (redes de conhecimento na aprendizagem STEM)

Artigos resumidos (<=3 páginas)

Estudos anteriores a 1991 (intervalo de 30 anos)

\section{Fonte: Autores (2021)}


O interesse dos pesquisadores estava apenas em estudos primários, publicados de 1991 até março de 2021, e que apresentassem alguma contribuição sobre os requisitos da pesquisa. $\mathrm{O}$ corpus da investigação foi formado por arquivos extraídos de sete bases de conhecimento, com suas respectivas contribuições: ACM Digital Library (6,9\%), Compendex Engineering Village (7,2\%), IEEE Xplore (2,0\%), ISI Web of Science $(0,7 \%)$, ScienceDirect Elsevier (18,5\%), Scopus (12,5\%) e SpringerLink (52,2\%), sendo depois importados no formato bibtex para um software auxiliar, especialista em revisão literária chamado Parsifal. No Gráfico 1, pode-se observar a tendência das publicações por ano.

Gráfico 1 - Curva de Tendência Anual de Artigos

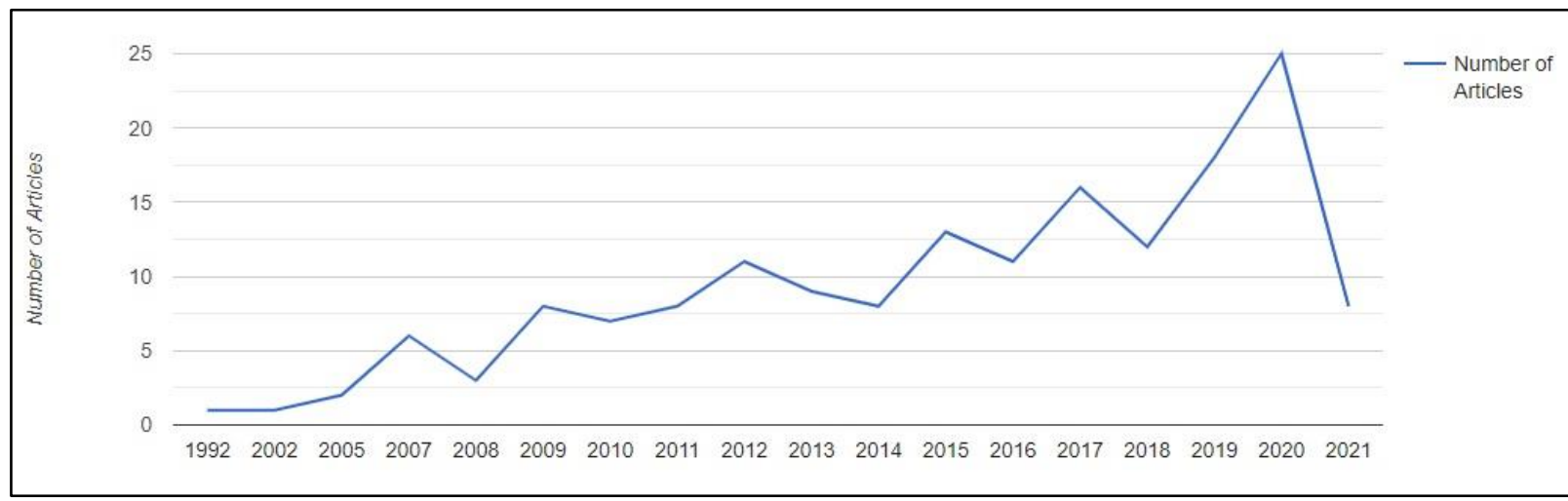

Fonte: Autores (2021)

Percebe-se uma alta constante de publicações desde 2018, chegando ao dobro da média em 2020. Quanto aos procedimentos de seleção de estudos, a Figura 1 apresenta as quatro etapas principais deste processo, incluindo as entradas e saídas de cada passo, com a quantidade de títulos.

Figura 1 - Etapas de Seleção de Estudos.

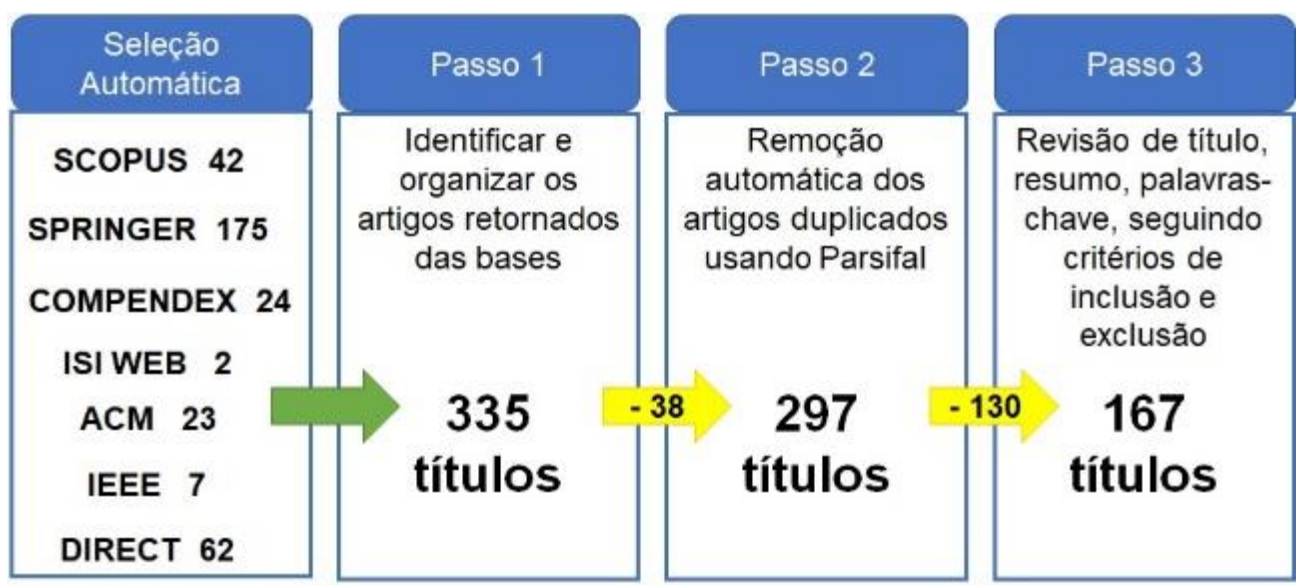

Fonte: Autores (2021)

Dando continuidade a fase de condução da SLR, entrou-se na etapa de avaliação da qualidade dos artigos aceitos, que é crítica, pelo fato de caracterizar-se pela distribuição de pesos de acordo com a importância dos estudos individuais, o que aumentou a acurácia dos resultados de extração de dados. Mas, ao mesmo tempo, alguns artigos com o índice (score) muito baixa foram excluídos do processo de análise e síntese. 
A avaliação de qualidade empregada na pesquisa procurou aplicar uma técnica de pontuação para avaliar a credibilidade, completude e relevância dos estudos selecionados. Neste ínterim, escolheu-se seis critérios de qualidade que englobaram dimensões de escrita, apresentação, metodológica e específica, conforme as diretrizes de Kitchenham e Charters (2007). O instrumento de avaliação utilizado é apresentado na Tabela 2:

Tabela 2 - Critérios de Avaliação da Qualidade da Pesquisa

\begin{tabular}{c|l}
\hline Número & \multicolumn{1}{c}{ Questão } \\
\hline Q1 & $\begin{array}{l}\text { O estudo busca compreender o processo de inovação por meio de } \\
\text { parâmetros observáveis e resultados claramente descritos? }\end{array}$ \\
\hline Q2 & $\begin{array}{l}\text { O estudo é realizado no contexto da educação profissional e } \\
\text { tecnológica? }\end{array}$ \\
\hline Q3 & O estudo conduz um experimento replicável para validar a proposta? \\
\hline Q4 & O objetivo da pesquisa está claramente descrito? \\
\hline Q5 & Os autores descrevem as limitações do estudo? \\
\hline Q6 & $\begin{array}{l}\text { O estudo fornece fundamentos teóricos para o termo "rede de } \\
\text { conhecimento"? }\end{array}$ \\
\hline
\end{tabular}

Fonte: Autores (2021)

Cada questão de avaliação da qualidade foi julgada em relação a três respostas possíveis: "Sim" (pontuação $=1$ ), "Parcialmente" (pontuação = 0,5 ) ou "Não" (pontuação = 0 ). Consequentemente, a pontuação da qualidade de um artigo em particular foi computada tomando a soma das pontuações das respostas as seis questões de avaliação, podendo chegar a uma escala de no máximo seis (6) e mínimo de zero (0) pontos. Após a avaliação, foi realizado um recorte na quantidade de artigos que foram submetidos ao Formulário de Extração de Dados. Somente os artigos que receberam uma pontuação máxima (6 pontos) passaram para uma etapa de análise mais completa do texto, conforme a Tabela 3:

Tabela 3 - Extrato de Artigos para Análise Completa

\begin{tabular}{l|c}
\hline \multicolumn{1}{c|}{ Título } & Pontos \\
\hline $\begin{array}{l}\text { Collaborative innovation: A study of creative teamwork in offshore industry and in design } \\
\text { education }\end{array}$ & 6 \\
\hline $\begin{array}{l}\text { Interorganisational social capital and innovation: a multiple case study in wine producers } \\
\text { networks in Serra Gaúcha }\end{array}$ & 6 \\
\hline $\begin{array}{l}\text { Cognitive strategies in solution mapping: How engineering designers identify problems } \\
\text { for technological solutions }\end{array}$ & 6 \\
\hline $\begin{array}{l}\text { Success Factors in Higher Education Industry Collaboration: A case study of } \\
\text { collaboration in the engineering field }\end{array}$ & 6 \\
\hline Emerging education hubs: the case of Singapore & 6 \\
\hline Proximity, knowledge integration and innovation: an agenda for agent-based studies & 6 \\
\hline $\begin{array}{l}\text { Envisioning engineering education and practice in the coming intelligence convergence } \\
\text { a complex adaptive systems approach }\end{array}$ & 6 \\
\hline $\begin{array}{l}\text { The growing complexity in invention process } \\
\text { Student customized creative education model based on open innovation }\end{array}$ & 6 \\
\hline The Knowledge Networks in a Makerspace: the Topologies of Collaboration & 6 \\
\hline Web-service architecture for tools supporting lifelong e-learning platforms & 6 \\
\hline
\end{tabular}




\begin{tabular}{l|c}
\hline Innovation spaces: New places for collective intelligence? & 6 \\
\hline Research and trends in the studies of collective intelligence from 2012 to 2015 & 6 \\
\hline $\begin{array}{l}\text { Knowledge management through the e-learning approach - a case study of online } \\
\text { engineering courses }\end{array}$ & 6 \\
\hline Collective inteligence in robotics labs: Mapping the flows of information & 6 \\
\hline
\end{tabular}

Fonte: Autores (2021)

Finalizando o protocolo da revisão sistemática da literatura, composta pelos componentes de questão de pesquisa, estratégia de busca e seleção dos estudos, instrumento de avaliação de qualidade, instrumento e procedimento de extração dos dados, passou-se para as etapas de estratégias de sumarização e análise dos resultados.

\subsection{Relatório da SLR}

O trabalho de escrita do relatório constitui este próprio artigo científico, portanto a descrição dos resultados e a discussão sobre as respostas das perguntas de pesquisa, levantadas no capítulo 3.1, foram registradas no capítulo que se segue.

\section{ANÁLISE E RESULTADOS DA PESQUISA}

A investigação coletou dados dos artigos em diferentes bases científicas, utilizando a estratégia e técnicas da metodologia de revisão sistemática da literatura ( $S R L)$, os quais são expostos nesta seção para discussão e resposta as indagações da pesquisa. A maioria dos artigos selecionados são do período de 2009 até 2020, sendo que o ano de 2017 representa $1 / 3$ do extrato textual. Entre os documentos, encontram-se pesquisas de universidades localizadas nos Estados Unidos, Reino Unido, Holanda, Noruega, Arábia Saudita, Coréia do Sul, Singapura e Brasil.

\subsection{O que é uma Rede de Conhecimento?}

O conceito de "Redes de Conhecimento" foi descrita como uma "inteligência coletiva" ou "capacidade humana de se envolver em cooperação intelectual a fim de criar, inovar e inventar" (GRIMON; MEZA; VACA-CARDENAS; MONGUET, 2017, MOREL; DUPONT; BOUDAREL, 2017). O que entra em consonância com a maioria dos autores ao empregarem a palavras "conexão" e "interação" nas suas definições. Completando a visão de que "Rede de Conhecimento" é uma "inteligência coletiva" - termo cunhado pelo filósofo francês Pierre Lévy (1994) - pode se dizer o seu produto seria uma "base de conhecimento", onde "os fluxos de informação são complexos" (BRAGA; GUTTMANN, 2019, BRAGA; SCHETTINI, 2019). Desta forma, pode-se afirmar que esta base de conhecimento, "mais ampla e diversa" (LUO; WOOD, 2017) forma uma espécie de "ecossistema de força cognitiva e de trabalho, capaz de enfrentar os desafios da indústria 4.0" (NOOR, 2013).

Segundo Pavel e Berg (2014), as vantagens das "Redes de Conhecimento" são o "empreendedorismo estratégico", e da mesma forma, a "inovação colaborativa". Isto é, seria um novo modus operandi do pensamento e da prática do engenheiro, que ao organizar o seu projeto, procura se arriscar mais, além do tradicional, com novas abordagens técnicas, científicas e tecnológicas, mas ao mesmo tempo reconhece seus limites e busca apoio e ampliação de suas ideias em sua comunidade de domínio. Na educação, esta mudança significa uma "sintonia com a prática profissional" (p. 6). 


\subsection{Como surge uma Rede de Conhecimento?}

Por causa da complexidade dos problemas e recursos diponíveis atualmente, as "Redes de Conhecimento" se transformam em um ambiente capaz de fomentar a criatividade, de uma educação convergente ao seu tempo, baseada na inovação aberta, na coconstrução de conhecimento e de trocas ativas por diferentes grupos (LUO; WOOD, 2017, KIM; RYOO; AHN, 2017). Há uma necessidade, por exemplo, de cooperação e reciprocidade entre os membros da área de engenharia, para reconhecimento de oportunidades de solução: "os projetistas de engenharia compartilham etapas de projetos e estratégias cognitivas comuns enquanto projetam com tecnologias e aplicações diferentes" (LEE; DALY; HUANG-SAAD; RODRIGUEZ; SEIFERT, 2020, p. 27). Então, as "Redes de Conhecimento" surgem "quando indivíduos fazem coisas coletivamente de forma inteligente" (GRIMON; MEZA; VACA-CARDENAS; MONGUET, 2017), onde este conjunto de "interações pode produzir um ambiente de aprendizagem cada vez melhor e continuamente aprimorado" (NOOR, 2013) e, portanto, esta "aplicação de recursos implica na formação de redes de conheciemento" (TAYLOR; MORONE, 2012).

O surgimento destas redes, conforme alguns autores, reforça o princípio da confiança para sua existência (BARABÁSI, 2009). Dentro desta teia de troca de informações, interesses e conflitos, se não existir a confiança mútua, sobraria apenas a competição pura, o que "levaria as empresas a reter o conhecimento dentro de seus limites e, assim, reduzir o grau de polinização cruzada cognitiva. Isso, por sua vez, reduziria a amplitude da inovação" (TAYLOR; MORONE, 2012, p. 2). A competência colaboração permeia toda a rede, do início ao fim, incluindo todos os seus membros, humanos e não humanos, pois muitas redes surgiram por causa de tecnologias interativas ou pelo simples impacto que alguns aparatos tecnológicos proporcionam em nossas formas de vida. Assim, todo processo de inteligência coletiva está sujeito as "interações humano-humano e homem-máquina no contexto do desenvolvimento ágil das empresas" (p. 7-8). Braga e Schettini (2019) corroboram para esta revelação, ao afirmarem que a introdução de artefatos no ambiente produtivo-colaborativo pode mudar a difusão da informação nas comunidades humanas.

\subsection{Quais são os objetivos de uma Rede de Conhecimento?}

Diante do que já foi exposto, pode-se dizer que um dos objetivos mais importantes das "Redes de Conhecimento" é a (i) formação para a inovação dentro dos programas educacionais (THUNE, 2010). Ainda, temos como objetivos importantes: (ii) a exploração de diferentes domínios, necessário para a tomada de decisão (GRIMON; MEZA; VACACARDENAS; MONGUET, 2017); (iii) fornecer uma estrutura de troca de conhecimento para a economia criativa e todos os atores envolvidos (KIM; RYOO; AHN, 2017); (iv) desenvolvimento de competências ao longo da vida (DIMOV; STEFANOV, 2009); (v) identificar caminhos e gargalos, visando melhorar a gestão do conhecimento nestes ambientes STEM (BRAGA; SCHETTINI, 2019).

\subsection{Que contribuições uma Rede de Conhecimento oferece para um processo de inovação?}

As "Redes de Conhecimento" necessitam de equipes multidisciplinares para processarem uma grande quantidade de informações de diferentes domínios. Neste sentido, são capazes de gerar modelos de inovação (THUNE, 2010), partindo de um modelo pedagógico que inclua a prática no trabalho de design colaborativo (PAVEL, BERG, 2014). Configurando assim, uma inovação aberta, rede formada pelas relações entre governo, universidades e empresas (KIM; RYOO; AHN, 2017). 
A inovação aberta pode ser compreendida pelo viés pedagógico, onde cria um "ambiente interativo para alunos, professores e engenheiros profissionais, onde o conhecimento pode ser construído e as experiências podem ser compartilhadas e trocadas" (AICHOUNI; BENCHICOU; NEHARI, 2013, p. 323). Mas, também pode-se olhar pelo viés produtivo, onde o aumento de capital social influencia a competitividade organizacional, que por consequência influenciam algumas dimensões do processo de inovação (FACCIN; GENARI; MACKE, 2017). Em resumo, as redes facilitam a criação de modelos STEM para o desenvolvimento tecnológico, mesmo que envolto a condições divergentes, econômicas e culturais, de seus membros (SIDHU; HO; YEOH, 2010).

A pesquisa trouxe à tona duas constatações: sobre a contribuição do conhecimento tácito para o processo de inovação e sobre a forma de se começar um processo de inovação. A primeira, corresponde a aprendizagem de técnicas para a criação de produtos, processos, ferramentas e gestão de pessoas, promovendo a inovação organizacional. Segundo estudos, as relações informais no intercâmbio de conhecimentos tácitos, possuem grande relevância (FACCIN; GENARI; MACKE, 2017). As experiências vividas por alguns membros da rede, quando compartilhadas, engrandecem todo o processo de criação e fabricação (BRAGA, GUTTMANN, 2019). Os autores reforçam este fato ao dizerem que "o conhecimento tácito não pode ser ensinado, pois só pode ser transmitido pela observação quando uma pessoa observa os outros fazendo uma atividade, dançando, tocando um instrumento, ou qualquer coisa que utilize habilidades desenvolvidas por ela mesma" (p. 2). Este tipo de conhecimento tácito, não circula com facilidade dentro das redes internas das organizações, como nos lembra Taylor e Morone (2012): "Isso se deve em grande parte ao fato de que o conhecimento relevante está cada vez mais fora da empresa" (p. 2).

O segundo ponto, trata-se de uma controvérsia. Enquanto alguns autores defendem que um projeto inovador começa pela solução, outros defendem que o primeiro passo de uma inovação é compreender bem um problema. Autores como o professor Jin Woo Lee, reconhecem o desenvolvimento tecnológico sem uma aplicação conhecida, como algo plausível, justamente pelo fato dos problemas complexos não serem de fácil compreensão, e lembra que redes de equipes abertas e ágeis podem desenvolver tecnologias de forma rápida e flexível: "[...] em alguns processos de projeto, um problema não é o primeiro foco; em vez disso, as soluções podem vir primeiro, como, por exemplo, quando novas tecnologias são criadas" (LEE; DALY; HUANG-SAAD; RODRIGUEZ; SEIFERT, 2020, p. 2). Em sua obra recente, Braga (2021) elucida que é preciso ter clareza do problema para que exista motivação na execução do projeto: "Existem casos em que equipes se propõem a elaboração de algo para o qual não sabem muito bem para que serve. Ao final, ficam tentando justificar de alguma forma a utilidade do artefato ou do processo" (p. 66). Segundo o autor, quando o problema é bem definido, as soluções serão mais diversificadas. E para expandir a controvérsia, alguns pesquisadores afirmam que o problema precisa ser "autêntico", isto é, ser um problema perceptível na realidade (HALLSTRÖM; SCHÖNBORN, 2019).

\subsection{Quais são as dificuldades comuns para se manter uma rede de conhecimento ativa?}

Entre as dificuldades apontadas pelos autores dos artigos selecionados destacamse: (i) a elaboração de estratégias específicas para para o mapeamento de soluções (LEE; DALY; HUANG-SAAD; RODRIGUEZ; SEIFERT, 2020); (ii) os projetos são simulações limitadas da realidade, não abrangendo todos os cenários e incertezas (PAVEL, BERG, 2014; LUO; WOOD, 2017, TAYLOR; MORONE, 2012); (iii) mudar antigas abordagens fechadas para novas mais abertas (KIM; RYOO; AHN, 2017). 


\section{Considerações Finais}

O trabalho em questão tenta contribuir com a problemática de formação de engenheiros e profissionais de tecnologia, que se aproxime mais das demandas e desafios da contemporaneidade. Verificou-se que aprender a trabalhar com um constante fluxo de informações e de forma colaborativa em rede, são requisitos para se aproximar teoria e prática, academia e setor produtivo. O quadro teórico reforçou o argumento de que as conexões e interações dos estudantes com a interdisciplinaridade dos saberes de forma prática pode criar caminhos de projetos inovadores, ou redes de conhecimento para a inovação colaborativa. Diante disto, levantou-se por meio da técnica de revisão sistemática da literatura, artigos nas principais bases científicas do mundo. Por fim o processo elencou 167 documentos, mas a etapa de validação da qualidade fez um corte nos artigos com nota mais alta, ficando o corpus textual com 15 artigos. A base destes artigos serviu para responder as perguntas da pesquisa. Para entender melhor o conceito de Redes de conhecimento, os autores sugeriram o termo "inteligência coletiva". O surgimento destas conexões deve se dar pelo que chamam de "inovação aberta", buscando as organizações na parte externa pelas informações que ainda não dispõem e principalmente pautando pelo Princípio da Confiança, onde a troca está baseada no ganha-ganha e não em simples competitividade. Como objetivos principais foram destacados a formação educacional para a inovação e uma aprendizagem ao longo da vida. As Redes de Conhecimento se configuram como um modelo de inovação, pois por meio delas o conhecimento tácito está mais presente e visível. Por fim, as dificuldades em mantê-las é que muitos projetos ainda carecem de simulações mais realistas para mensurar as incertezas.

Sendo assim, a pesquisa dá mais um passo para esclarecer o conceito de Redes de Conhecimento e como trabalhos futuros pode-se aumentar o corpus textual para ampliar a gama de autores e percepções sobre o tema.

\section{REFERÊNCIAS}

BARABÁSI, A. Linked: a nova ciência dos networks. Trad. Jonas P. dos Santos. São Paulo: Leopardo Editora, 2009.

BRAGA, M. Aprendendo a inovar em projetos STEAM: um guia para estudantes. Rio de Janeiro: E-papers, 2021.

CAMPOS, L., et al.: Desafios da Educação em Engenharia: vocação, formação, exercício profissional, experiências metodológicas e proposições. In: XXXIX Congresso Brasileiro de Educação em Engenharia. Anais... Blumenau, SC, Brasil, 2011, p. 113-164.

CAVALCANTE, L.; VALENTIM, M. Comportamento informacional em ambientes empresariais. In: VALENTIM, M. (Org.). Gestão da informação e do conhecimento no âmbito da Ciência da Informação. São Paulo: Polis/Cultura Acadêmica, 2008. p. 117-128.

Confederação Nacional da Indústria ( $\mathrm{CNI})$. Desafios para a indústria 4.0 no Brasil. Brasília: CNI, 2016. 
GODOY, E.; ALMEIDA, E. Evasão nos cursos de engenharia: um olhar para os trabalhos do COBENGE de 2000 a 2014. Revista Brasileira de Ensino de Ciência e Tecnologia, Ponta Grossa, v.13, n. 3, p. 50-74, set./dez. 2020.

HALLSTRÖM, J., SCHÖNBORN, K.J. Models and modelling for authentic STEM education: reinforcing the argument. IJ STEM, v. 6, n. 22, 2019.

LIMA, C.; REIS, A.; SILVA, J.; SILVA, S.; FUJIHARA, Y., CARVALHO, C. A importância de atividades práticas para o desenvolvimento profissional do engenheiro. In: XLVIII COONGRESSO BRASILEIRO DE EDUCAÇÃO EM ENGENHARIA, 2020, [evento online]. Anais... Caxias do Sul: ABENGE/UCS, 2020. Disponível em: <https://doi.org/10.37702/COBENGE.2020.3146>. Acesso em: 12 jan. 2020.

KITCHENHAM, B.; CHARTERS, S. Guidelines for performing systematic literature reviews in software engineering. Technical Report EBSE 2007-001, Keele University and Durham University Joint Report, 2007.

MORAES, C.; FADEL, B. Ambiência organizacional, gestão da informação e tecnologia. In: VALENTIM, M. (Org.). Informação, conhecimento e inteligência organizacional. 2. ed. Marília: FUNDEPE Editora, 2007. p. 99-114.

OKOLI, Chitu. A guide to conducting a standalone systematic literature review. Communications of the Association for Information Systems, v. 37, n. 43, p. 879-910, nov. 2015. Disponível em: http://aisel.aisnet.org/cais/vol37/iss1/43/. Acesso em: 30 jan. 2021.

SÁNCHEZ LUDEÑA, E. La educación STEAM y la cultura «maker». Padres y Maestros / Journal of Parents and Teachers, n. 379, p. 45-51, set. 2019.

TAN, S; CHAN, C.; BIELACZYC, K. et al. Knowledge building: aligning education with needs for knowledge creation in the digital age. Education Tech Research Dev, 2021.

TONINI, A.; PEREIRA, T. (Orgs.) Desafios da Educação em Engenharia: Empreendedorismo, Industria 4.0, Formação do Engenheiro, Mulheres em STEM. Brasília: ABENGE, 2019.

THOMASSEN, A.; STENTOFT, D. Educating Students for a Complex Future: Why Integrating a Problem Analysis in Problem-Based Learning Has Something to Offer. The Interdisciplinary Journal of Problem-based Learning - IJPBL, v. 4, n. 2, set. 2020.

VEDENPÄÄ, I.; LONKA, K. Teachers' and Teacher Students' Conceptions of Learning and Creativity. Creative Education, n. 5, 2014, p. 1821-1833.

WORLD ECONOMIC FORUM. Jobs of Tomorrow: Mapping Opportunity in the New Economy. Davos, 2020. Disponível em: <http://www3.weforum.org/docs/WEF_Jobs_of_Tomorrow_2020.pdf>. Acesso em: 24 fev. 2021. 


\title{
STEAM KNOWLEDGE NETWORKS IN CONTEMPORARY ENGINEERING EDUCATION: A SYSTEMATIC REVIEW OF THE LITERATURE
}

\begin{abstract}
This paper shows the difficulty of finding STEM learning models that enhance information flow and networking for innovation. Then, it was intended to elucidate about the concept and creation of Knowledge Networks. The research methodology used was a systematic literature review, which collected 167 articles from the main scientific bases to support the discussion. The quality validation step cut out 15 articles to answer the research questions. The results present the Knowledge Networks as ecosystems of collective intelligence, which arise mostly as a response to competitiveness and collaboration. Their main objective is training for innovation.
\end{abstract}

Keywords: STEM education, Knowledge Networks, Collaborative Innovation, Projectbased Learning 